\title{
Evolving Geometric Phase and Its Dynamical Manifestation as a Frequency Shift: An Optical Experiment
}

\author{
R. Simon \\ The Institute of Mathematical Sciences, Madras 600113 , India \\ and \\ H. J. Kimble and E. C. G. Sudarshan \\ Department of Physics, University of Texas at Austin, Austin, Texas 78712 \\ (Received 25 January 1988)
}

\begin{abstract}
We show that an evolving geometric phase induces a frequency shift. We report an optical experiment where we employ this effect to offset the frequency of a laser beam.

PACS numbers: 03.65. Bz, 42.10.Jd, 42.10.Nh, 42.50.Wm
\end{abstract}

A system (classical or quantum) undergoing periodic motion has a dynamical phase which is the time integral of the frequency. If the cyclic evolution takes place in a slowly varying environment, then there is an additional nondynamical phase that is associated with the closed circuit that the system traces in the projective Hilbert space of states. In a series of papers, Berry ${ }^{1}$ has shown how this nondynamical phase may be understood geometrically in the case of a cyclic adiabatic evolution of parameters in the system Hamiltonian, with the state of the system being a discrete nondegenerate eigenstate of the Hamiltonian. The geometry underlying Berry's phase was clarified by Simon, ${ }^{2}$ who reformulated it in terms of a line bundle. More recently, Ramaseshan and Nityananda ${ }^{3}$ have pointed out that a phase discovered by Pancharatnam ${ }^{4}$ in the context of polarized light may be interpreted as an early example of Berry's phase.

Anandan and Stodolsky ${ }^{5}$ studied Berry's phase for a collection of states and showed them to be related to the composition of finite elements in a Lie group. Further, Aharanov and Anandan ${ }^{6}$ have liberated the geometric phase from the restriction to adiabatic evolutions; they have given a formula in terms of the closed circuit of the state vector in the projective Hilbert space. In the special case of these evolutions being brought about by adiabatic parameter variations, their results agree with those of Berry.

We shall be concerned here with a more general question: What if the geometric phase itself is evolving? This can come about, for example, in the following ways: (1) by repetitive traversals of the circuit in the projective space, or (2) by continuous deformation of the circuit. In these cases the result is a phase modulation resulting generically in a spectral broadening. ${ }^{7}$ But in the special case where the geometric phase $\beta$ evolves linearly in time, the constant $d \beta / d t$ leads to a change in the frequency. In the case where the time evolution of $\beta$ is brought about by a continuous deformation of the circuit it is understood that the time scale that characterizes this deformation is large compared to the time of traversal of the circuit so that the quasistatic approximation becomes appropriate and the geometric phase at any time can be computed with the instantaneous circuit. We report here an experiment wherein we have observed such a shift in frequency.

The experiment involves unitary transformations on the polarization state of a light beam. ${ }^{3,4}$ It will be found that the generator of the transformations is piecewise constant, making the evolution nonadiabatic. Thus, the Aharonov-Anandan ${ }^{6}$ framework in terms of a closed circuit in the projective Hilbert space rather than the parameter space forms a natural setup for analysis of the experiment. The dynamical phase is the integral of the expectation value of the generator of the unitary transformation, while the geometric phase depends only on the closed circuit in the projective Hilbert space; the total phase is the sum of these two phases.

The polarization of a plane light wave propagating along the $z$ direction is defined by a pair of complex electric-field components $E_{x}, E_{y}$ arranged as a column vector $E$. The projective space of distinct polarization states is defined by the ratio $E_{x} / E_{y}$ with the points at infinity identified, and thus is isomorphic to a sphere $S^{2}$ called the Poincare sphere ${ }^{4}$ shown in Fig. 1. The north and south poles represent right and left circular polarizations and the points in the equatoral plane describe linear polarizations. Orthogonal states are represented by diametrically opposite points.

We shall be interested in intensity-preserving linear optical transformations which act on $E_{x}, E_{y}$ by $2 \times 2$ unitary matrices constituting the group U(2); they act on the Poincaré sphere by rotations. In particular a quarter-wave plate acts as a rotation through $\pi / 2$ about an axis in the equatorial plane of the Poincare sphere; if the slow axis of the quarter-wave plate is along the $x$ direction, then the rotation on the Poincare sphere is about $O A$. A rotation of the quarter-wave plate (about the $z$ axis normal to the plate) by $\theta$ would rotate this 


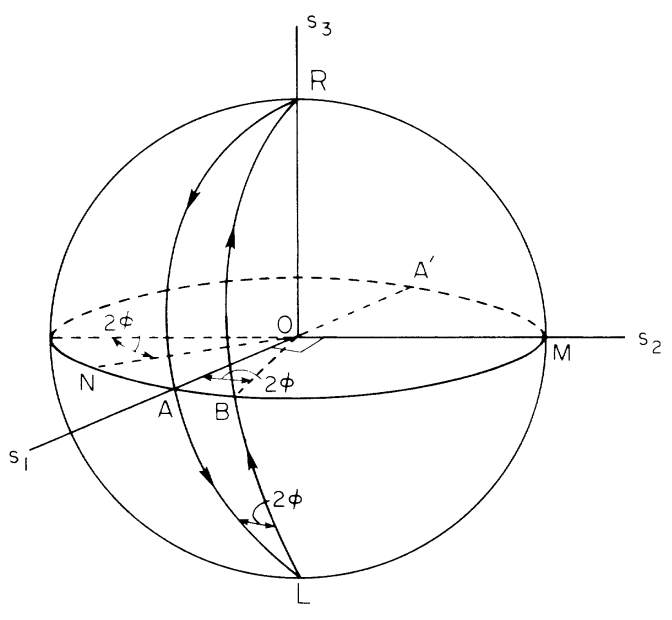

FIG. 1. Distinct polarization states are shown on the Poincaré sphere. The polar points $\mathrm{R}, \mathrm{L}$ represent right and left circular polarizations. Linear polarization along $x$ is represented by $\mathrm{A}$ and the orthogonal $y$ polarization by $\mathrm{A}^{\prime}$. The first quarter-wave plate produces a $\pi / 2$ rotation with $O M$ as the axis, and the second one with $O N$ as the axis.

equatorial axis by $2 \theta$.

Consider light polarized along the $x$ axis incident on a quarter-wave plate whose slow axis makes an angle of $\pi / 4$ with the $x$ axis. The emergent light which is left circularly polarized then passes through a second quarterwave plate whose slow axis makes an angle of $3 \pi / 4+\phi$ with the $x$ axis. The incident light is represented by the point $\mathrm{A}$ on the Poincare sphere, the light emerging from the first quarter-wave plate by $\mathrm{L}$, and that from the second quarter-wave plate by the linear polarization state B. This light falls normally on a plane mirror and after reflection passes back through the two quarterwave plates. The polarization state is transformed from $\mathrm{B}$ to $\mathrm{R}$ and then to $\mathrm{A}$. Clearly, the unitary evolution $A L B R A$ is cyclic for every $\phi$. For $y$-polarized light the evolution is again cyclic, but given by the reflection of this circuit through the origin. Clearly, the two circuits are traversed in opposite senses; and hence subtend equal and opposite solid angles at the origin. The solid angle subtended by the circuit $A L B R A$ is $4 \phi$. Since these are SU(2) transformations the Berry phase is half the solid angle. Thus $x$ and $y$ polarizations develop Berry phases of $+2 \phi$ and $-2 \phi$ under this sequence of transformations.

Now, if the second quarter-wave plate is rotated uniformly with an angular velocity $\Omega=d \phi / d t$, the segment $R A L$ of the circuit remains unaltered under this rotation, whereas the segment $L B R$ describing the action of the second quarter-wave plate rotates about the axis $R L$ with an angular velocity $2 \Omega$. Hence, the geometric phase changes linearly in time, $\beta(t)=\beta(0) \pm 2 \Omega t$, where the upper and lower signs are for $x$ and $y$ polarizations respectively, and therefore contributes a shift in frequency.

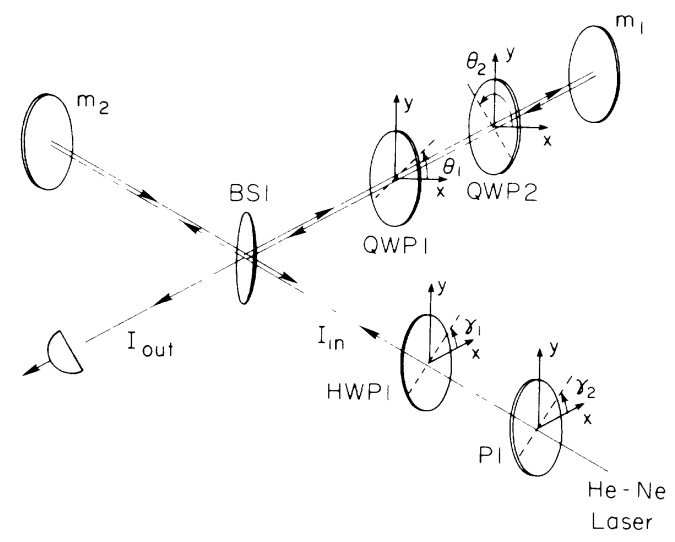

FIG. 2. Experimental arrangement for the generation of a time-varying geometric phase. The basic configuration is that of a Michelson interferometer, with the light from arm $m_{2}$ serving as a reference beam for the detection of phase changes generated in arm $m_{1}$. QWP1 is a quarter-wave plate with its slow axis fixed at $\theta_{1}=\pi / 4$. The slow axis of quarter-wave plate QWP2 is slowly rotated about the propagation direction with angular velocity $\Omega=d \theta_{2} / d t=d \phi / d t$. The incident beam is linearly polarized along $x$ or $y$.

If the input light was of frequency $\omega$, the output light (after making the round trip) will have frequency $\omega^{\prime}=\omega \mp 2 \Omega$, where we note that a positive geometric phase corresponds to a phase retardation and the minus (plus) sign is for $x(y)$ polarization. In evaluating this evolving geometric phase $\beta(t)$, we assume that $\Omega^{-1}$ is very large compared to the time of transversal of the circuit so that $\beta(t)$ is related to the solid angle subtended by the instantaneous circuit.

The experimental arrangement that we have employed to observe this frequency shift is illustrated in Fig. 2. The apparatus is a Michelson interferometer with a time-varying phase shift generated in one arm $\left(\mathrm{m}_{1}\right)$ by quarter-wave plates QWP1 and QWP2 in double pass precisely as in the previous discussion. The slow axis of QWP1 is fixed at angle $\theta_{1}=\pi / 4$ relative to the $x$ axis while that of QWP2 is specified by an angle $\theta_{2}$. QWP2 is rotated about the $z$ axis with an approximately constant angular velocity $\Omega=d \theta_{2} / d t$. Apart from a constant offset, $\theta_{2}$ is just $\phi$ as illustrated in Fig. 1. The second arm of the interferometer $\left(\mathrm{m}_{2}\right)$ provides a reference beam against which phase changes due to the rotation can be measured. The orientation of the axis of the linear polarization state of the input field is controlled with half-wave plate HWP1. The input beam is from a single-frequency helium-neon laser operating at $\lambda=633$ $\mathrm{nm}$.

One of the most important aspects of any experiment dealing with topological phase is the need to ensure that the dynamical phase remains constant while the parameters that lead to the topological phase are changed, so 


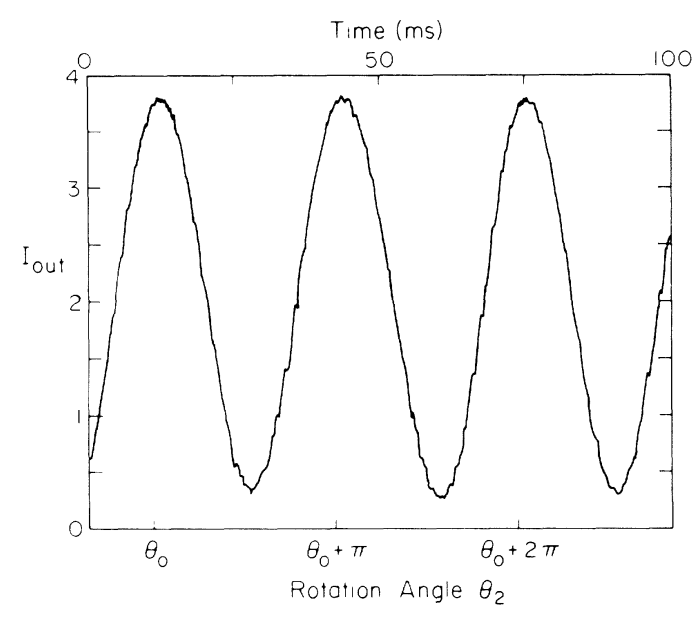

FIG. 3. Variation of the transmitted intensity $I_{\text {out }}$ as a function of the rotation angle $\theta_{2}$ of QWP1, with $\theta_{2}$ defined in Fig. 2. The approximately periodic variation in $I_{\text {out }}$ is a result of a beat note between the fields from the two arms $\left(\mathrm{m}_{1}, \mathrm{~m}_{2}\right)$ of the interferometer, with the frequency shift $\Delta \omega=-200 / \mathrm{s}$ produced by a linearly increasing topological phase.

that the measured change in phase can be identified with the change in the topological phase. Thus, in the present setup we have to show that the dynamical phase is independent of $\theta_{2}$ (or equivalently of $\phi$ ). To this end first note that the evolution in $z$ of the polarization state vector $E$ is described by $\lambda d E / d z=i J(z) E$, where the generator $J(z)$ is a $2 \times 2$ Hermitian matrix whose elements are dimensionless, and $\lambda=\lambda / 2 \pi{ }^{8}$ By a comparison of this relation with Eq. (2) of Ref. 6, it is seen that $z$ plays the role of $t$ and $\lambda$ that of $\hbar$. For free propagation $J(z)$ is the identity matrix, and for a birefringent plate whose

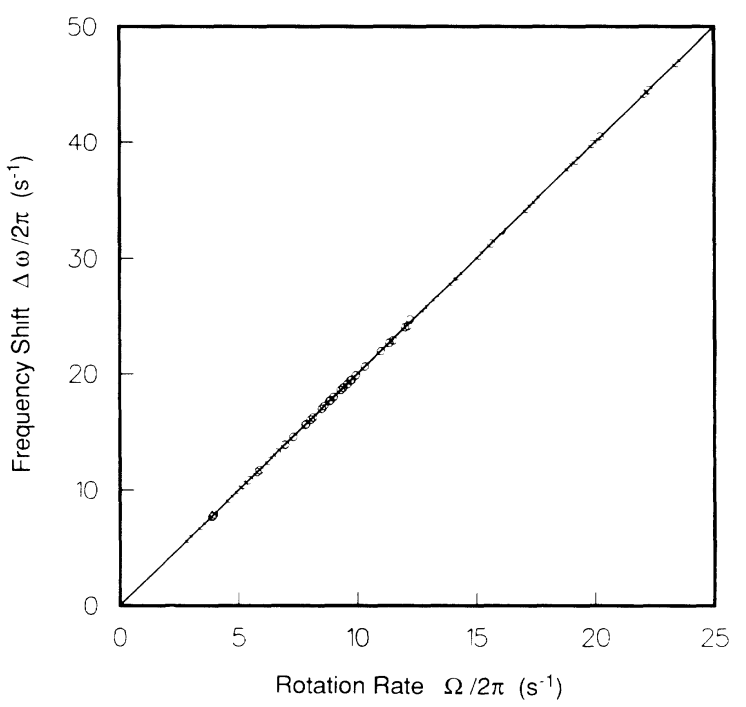

FIG. 4. Modulus of the frequency shift $\Delta \omega$ vs rotation rate $\Omega$. For each measured rotation rate, a spectral analysis of the photocurrent from $I_{\text {out }}$ gives the frequency shift between the beams from the two arms of the interferometer. The straight line is the theoretical result $|\Delta \omega|=2 \Omega$.

slow axis makes angle $\theta$ with the $x$ axis

$$
J_{\theta}(z)=J_{\theta}=\left(\begin{array}{cc}
n+\epsilon \cos 2 \theta & \epsilon \sin 2 \theta \\
\epsilon \sin 2 \theta & n-\epsilon \cos 2 \theta
\end{array}\right),
$$

where $n+\epsilon$ and $n-\epsilon$ are the refractive indices for the slow and fast components. For a quarter-wave plate, the thickness $d$ is such that $2 \epsilon d \cong \lambda / 4(\bmod \lambda)$. The dynamical phase introduced by the birefringent plate is, if we assume $E^{\dagger} E=1$ and use Eq. (3) of Ref. 6,

$$
(d / \chi) E^{\dagger} J_{\theta} E=(d / \chi)\left[n+\epsilon \cos 2 \theta\left(\left|E_{x}\right|^{2}-\left|E_{y}\right|^{2}\right)+\epsilon \sin 2 \theta\left(E_{x} E_{y}^{*}+E_{y} E_{x}^{*}\right)\right] .
$$

From Eq. (2) it is clear that for circularly polarized light the dynamical phase introduced by a quarter-wave plate is independent of $\theta$. In all the four encounters of the quarter-wave plates in our experiment, the incident light is either circularly polarized or linearly polarized at $\pm 45^{\circ}$ to the slow axis, and hence the dynamical phase introduced by the wave plates for the circuit of Fig. 1 is $4 n d / \lambda$, independent of $\theta_{2}$. The invariance of the dynamical phase under change in $\theta_{2}$ is a particular case of a more general result that can be proved. Since every $J(z)$ corresponds to a rotation on the Poincaré sphere about some axis, a state represented by a point on the great circle perpendicular to this axis moves on this great circle, and for this state the dynamical phase is completely fixed by the $U(1)$ part (trace) of the $U(2)$ generator $J(z)$ and the geometric phase depends only on the SU(2) part of $J(z)$. Thus, if we have a sequence of wave plates taking a state over a spherical polygon, rotating the various plates to obtain a different spherical polygon will change only the geometric phase leaving the

\section{dynamical phase invariant.}

From this discussion we see that as the second quarter-wave plate is rotated the dynamical phase does not vary, but the topological phase varies linearly in $\theta_{2}$. Further, as the phase difference $\psi$ for propagation along the two arms of the interferometer is changed, the intensity $I_{\text {out }}$ of the transmitted superposition beam likewise varies, with $I_{\text {out }}=\alpha I_{\text {in }}(1+v \cos \psi)$. Here $v$ is the fringe visibility and $\alpha$ is related to the losses in propagation through the interferometer. For an initial polarization state along $x$, the transformations depicted in Fig. 1 are made successively at the elements QWP1 and QWP2 in the $\mathrm{m}_{1}$ arm of the interferometer. The phase retardation $\psi$ resulting from these transformations is given by $\psi=\psi_{0}+2 \theta_{2}$, where $\psi_{0}$ is a constant independent of the orientation of QWP2. For the case of a rotating wave plate QWP2, $\theta_{2}=\Omega t$ and the frequency shift is $\omega^{\prime}-\omega$ $\equiv \Delta \omega=-d \beta / d t=-2 \Omega$. The intensity of the transmitted field $I_{\text {out }}$ thus should oscillate in time as $I_{\text {out }}$ 
$=\alpha I_{\text {in }}\left[1+v \cos \left(\psi_{0}+2 \Omega t\right)\right]$.

This simple prediction of a phase retardation $\psi$ which is linearly increasing at twice the rotation rate is confirmed by the measurements shown in Figs. 3 and 4 . From Fig. 3 we see that the quantity $I_{\text {out }}$ exhibits a simple sinusoidal dependence (apart from the jitter associated with the unfortunate mechanical instability of the interferometer) as a function of time or of rotation angle $\theta_{2}$. The change $\Delta \psi=\Delta \beta=2 \pi$ is accomplished by a physical rotation of QWP2 of $\Delta \theta_{2}=\Delta \phi=\pi$, as expected. That the phase difference $\psi$ is linearly increasing in time is a reflection of a frequency shift between the two fields emerging from the arms of the interferometer. From Fourier analysis of time series such as that in Fig. 3, we can obtain the frequency shift $\Delta \omega$ as a function of rotation rate $\Omega$, as shown in Fig. 4. The relation $|\Delta \omega|=2 \Omega$ is well confirmed by the data in this figure, which are taken for incident polarizations along both $x$ and $y$ and for angles $\theta_{1}=\pi / 4$ and $3 \pi / 4$. That the signs of the respective phase changes and frequency shifts are in agreement with our analysis is confirmed by our scanning $m_{1}$ to produce either a phase advance or retardation for the $m_{1}$ arm relative to the $m_{2}$ arm. We have also generated frequency shifts of $4 \Omega$ between two orthogonally polarized beams emerging from $\mathrm{BS} 1$ by injecting a single beam polarized along the optic axis of QWP1.

In summary, we have in this Letter explored and verified a new kind of effect: In addition to the frequency's inducing a dynamical phase, the evolving nondynamical geometric phase induces a frequency shift. Since $\Omega$, the angular frequency of the second compensator, can be varied in a controlled manner, it follows that our arrangement employs Berry's phase to give a method for fine tuning of the frequency of a laser beam. In fact this method has been previously demonstrated in a different context for the generation of single-sideband carrier modulation. ${ }^{9}$ The cycle of transformations of Fig. 1 involving the polarization state is a sequence of SU(2) transformations; therefore, the Berry phase is half the solid angle subtended by the closed circuit at the origin, even though the photon has unit "spin." The Tomita and Chiao experiment ${ }^{10}$ involved a sequence of SO(3) transformations on the propagation direction with fixed polarization (helicity); hence, in contrast, a Berry's phase equal to the solid angle was obtained.

The polarization of plane waves and their transformation by quarter-wave plates are conventionally well described in classical terms; this is the perspective that we have adopted in this Letter. An earlier optical experi- ment ${ }^{10}$ related to Berry's phase has generated a lively discussion as to whether the effect is classical or quantum mechanical. ${ }^{11}$ From the work of Berry ${ }^{1}$ and of Anandan and Stodolsky ${ }^{5}$ it is clear that the Berry phase depends upon the sequence of unitary transformations, which manifest themselves as mode transformations in the quantum-field-theoretic description. Since in all optical experiments reported thus far, manifestations of the Berry phase are measured via the mutual coherence function, the classical and quantum treatments must give identical results, as demanded by the first fundamental theorem of quantum optics. ${ }^{12}$

One of the authors (R.S.) acknowledges the excellent hospitality of the University of Texas at Austin where part of this work was completed. We are grateful to J. L. Hall for bringing the work in Ref. 9 to our attention. Our work was supported by the Department of Energy under Grant No. DE-FG05-85ER40200 and by the Venture Research Unit of British Petroleum America.

${ }^{1}$ M. V. Berry, Proc. Roy. Soc. London A 392, 45 (1984), and J. Phys. A 18, 15 (1985), and Nature (London) 326, 277 (1987), and Proc. Roy. Soc. London A 414, 31 (1987).

${ }^{2}$ B. Simon, Phys. Rev. Lett. 51, 2167 (1983).

${ }^{3}$ S. Ramaseshan and R. Nityananda, Curr. Sci. 55, 1225 (1986); R. Bhandari and J. Samuel, Phys. Rev. Lett. 60, 1211 (1988); T. H. Chyba, L. J. Wang, L. Mandel, and R. Simon, Opt. Lett. (to be published).

${ }^{4}$ S. Pancharatnam, Proc. Indian Acad. Sci. Sect. A 44, 247 (1956), reprinted in Collected Works of S. Pancharatnam (Oxford Univ. Press, London, 1975).

5 J. Anandan and L. Stodolsky, Phys. Rev. D 35, 2597 (1987).

${ }^{6}$ Y. Aharonov and J. Anandan, Phys. Rev. Lett. 58, 1593 (1987).

${ }^{7}$ R. Simon and N. Kumar, J. Phys. A (to be published).

${ }^{8}$ The similarity between this equation and the Schrödinger equation for a two-level system is worth noting. Just as the Berry phase is consistent with and contained in the Schrödinger description, so also is the topological phase in polarization experiments consistent with the Jones calculus as is easily confirmed by multiplication of the Jones matrices for the individual transformations.

${ }^{9}$ C. F. Buhrer, D. Baird, and E. M. Conwell, Appl. Phys. Lett. 1, 46 (1962).

${ }^{10}$ A. Tomita and R. Y. Chiao, Phys. Rev. Lett. 57, 937 (1986).

${ }^{11}$ F. D. M. Haldane, Phys. Rev. Lett. 59, 1788 (1987).

${ }^{12}$ E. C. G. Sudarshan, J. Math. Phys. Sci. 3, 121 (1969). 